\title{
Qualidade fisiológica de sementes de Eruca sativa L. pelo teste de deterioração controlada
}

\author{
Physiological quality of Eruca sativa L. seeds by the test of controlled deterioration
}

\author{
Charline Zaratin Alves $^{\mathrm{I}^{*}}$ Amanda Regina Godoy ${ }^{\mathrm{I}}$ Ana Carina da Silva Candido \\ Marco Eustáquio de Sán
}

\section{RESUMO}

A qualidade da semente utilizada no processo de produção agrícola é um dos principais fatores a ser considerado para a implantação da cultura, havendo consenso entre todos os segmentos, sobre a importância do vigor das sementes e da necessidade de avaliá-lo. O presente trabalho teve o objetivo de estudar variações na condução do teste de deterioração controlada, verificando sua eficiência na identificação de diferentes níveis de vigor de sementes de rúcula, cultivar 'Cultivada' e 'Gigante' utilizando-se cinco lotes de sementes para cada um. As sementes foram submetidas à determinação do teor de água e a testes de germinação, primeira contagem de germinação, emergência e, para verificar a eficiência na identificação de diferentes níveis de vigor, foram estudadas variações no teste de deterioração controlada (teor de água de 18, 21 e $24 \%$, a $45^{\circ} \mathrm{C}$ durante 24 e 48 horas). $O$ experimento foi montado no delineamento inteiramente casualizado e os resultados foram submetidos à análise de variância, sendo que as médias foram comparadas pelo teste de Tukey a 5\% de probabilidade. Pelos resultados obtidos no teste de deterioração controlada, observou-se que não houve relação com as avaliações iniciais do potencial fisiológico das sementes, principalmente para o teste de emergência. Concluiu-se que, nas condições estudadas, esse teste não é sensível o suficiente para a avaliação do potencial fisiológico de sementes de rúcula, para ambas as cultivares estudadas.

Palavras-chave: rúcula, hortaliça, potencial fisiológico.

\section{ABSTRACT}

The quality of the seed used in the process of agricultural production is one of the main factors to be considered for the implantation of the culture, having consensus between all the segments, on the importance of the vigor of the seeds and the necessity to evaluate it. This research had the purpose of studying proccedings for controlled deterioration test, to verify their sensitivity for identifying different vigor levels of arugula seed, cv. 'Cultivada' and 'Gigante', using five lots of seeds for each. The seeds were subjected to the determination of water content and tests of germination, first germination, emergence, and to verify the efficiency in identifying different levels of vigor, were studied variations in the controlled deterioration test (seed moisture content of 18, 21 and 24\%, at $45^{\circ} \mathrm{C}$ for 24 and 48 hours). The experiment was arranged in completely randomized design and the results were submitted to analysis of variance and means were compared by Tukey test at $5 \%$ probability. The results obtained for the controlled deterioration test showed that there was no relation with the initial assessments of the physiological potential, especially for testing of emergency. It was concluded that under the conditions tested, this test was not sensitive enough to assess the potential physiologic arugula, for both cultivars.

Key words: arugula, vegetable, potential physiological.

\section{INTRODUÇÃO}

A rúcula (Eruca sativa L.) é originária da região mediterrânea, onde é conhecida desde a antiguidade, sendo que o primeiro registro data do século I, encontrado no herbário Grego Dioscorides. Na Itália, essa hortaliça folhosa é consumida em larga escala, sendo apreciada pela sua pungência, enquanto no Brasil é consumida na forma de salada crua e em

'Departamento de Agronomia, Universidade Federal de Mato Grosso do Sul (UFMS), 79560-000, Chapadão do Sul, MS, Brasil. E-mail:charline.alves@ufms.br. *Autor para correspondência.

"Departamento de Fitotecnia, Tecnologia de Alimentos e Socioeconomia, Universidade Estadual Paulista (UNESP), Ilha Solteira, SP, Brasil. 
pizzas, sendo que, nos últimos anos, houve um aumento na sua popularidade e consumo (PURQUERIO, 2005). As sementes, devido ao alto valor comercial, merecem atenção especial quanto ao potencial fisiológico, entretanto, há uma demanda por informações que possam fornecer subsídios e apoio aos programas de controle de qualidade.

O teste de deterioração controlada emprega uma técnica similar a do envelhecimento acelerado, incorporando um maior controle do teor inicial de água da semente durante o período de deterioração. Nesse teste, o teor de água das sementes é submetido a um mesmo nível, em todas as amostras, antes do início do período de deterioração (MATTHEWS, 1980; HAMPTON \& TEKRONY, 1995). Assim sendo, o teste de deterioração controlada revela-se promissor na separação de lotes de sementes com distintos níveis de vigor e, em função de sua eficiência, merece atenção dos tecnologistas de sementes.

Por se tratar de um procedimento relativamente simples, porém minucioso, esse teste tem sido utilizado para detectar diferenças no vigor de lotes de sementes, principalmente as de tamanho menor e para verificar o potencial de armazenamento de sementes de diversas hortaliças, tais como cenoura, cebola, alface e brássicas (MATTHEWS, 1980; POWELL \& MATTHEWS, 1981). No Brasil, diversos autores observaram resultados consistentes para avaliação do vigor de sementes de hortaliças, tais como pimentão (PANOBIANCO \& MARCOS FILHO, 1998), tomate (RODO et al., 1998; PANOBIANCO \& MARCOS FILHO, 2001a) e brócolis (SADER et al., 2001).

Pelos resultados do teste de deterioração controlada, tem-se verificado alta relação dentro e entre diferentes laboratórios (POWELL et al., 1984), como também alta correlação com a emergência de plântulas em campo para diversas espécies como: cebola, alface, nabo, beterraba, cenoura (MATTHEWS \& POWELL, 1987) e brócolis (MENDONÇA et al., 2000). O potencial de armazenamento de lotes de sementes também pode ser previsto mediante a utilização do teste de deterioração controlada, conforme resultados obtidos por POWELL et al. (1984), para sementes de cebola e couve de bruxelas.

Na literatura, há relatos de que o teste de deterioração controlada tem sido amplamente empregado utilizando sementes com teor de água, tempo de exposição e temperatura variáveis, como, por exemplo, alface, couve-flor e couve de bruxelas, $20 \% /$ $45^{\circ} \mathrm{C} / 24 \mathrm{~h}$ (ISTA, 1995 ); berinjela, $24 \% / 41^{\circ} \mathrm{C} / 48 \mathrm{~h}$ (BHERING et al., 2001a); beterraba e cenoura, 24\%/ $45^{\circ} \mathrm{C} / 24 \mathrm{~h}$ (ISTA, 1995); brócolis, $22 \% / 40^{\circ} \mathrm{C} / 24 \mathrm{~h}$ (MENDONÇA et al., 2000); ervilha, $20 \% / 45^{\circ} \mathrm{C} / 24 \mathrm{~h}$ (POWELLet al., 1997); maxixe, $19 \% / 45^{\circ} \mathrm{C} / 24 \mathrm{~h}$ (TORRES et al., 1998); melancia, $24 \% / 41^{\circ} \mathrm{C} / 48 \mathrm{~h}$ (BHERING et al., 2001b); melão, $21 \% / 45^{\circ} \mathrm{C} / 72 \mathrm{~h}$ (OLUOCH\& WELBAUM, 1996); nabo, $20 \% / 45^{\circ} \mathrm{C} / 24 \mathrm{~h}$ (ZHANG \& HAMPTON,
1999); pepino, $24 \% / 45^{\circ} \mathrm{C} / 48 \mathrm{~h}$ (BHERING et al., 2000); pimentão, $24 \% / 45^{\circ} \mathrm{C} / 24 \mathrm{~h}$ (PANOBIANCO \& MARCOS FILHO, 1998); repolho, $24 \% / 45^{\circ} \mathrm{C} / 24 \mathrm{~h}$ (STRYDOM \& VAN DE VENTER, 1998) e tomate, $24 \% / 45^{\circ} \mathrm{C} / 24 \mathrm{~h}$ (PANOBIANCO \& MARCOS FILHO, 2001a). Porém, para sementes de rúcula, ainda não existe um padrão definido para esse teste.

Nesse sentido, o objetivo deste trabalho foi estudar variações na condução do teste de deterioração controlada, verificando sua eficiência na identificação de diferentes níveis de vigor de lotes de sementes de rúcula.

\section{MATERIAL E MÉTODOS}

O trabalho foi conduzido no Laboratório de Tecnologia de Sementes do Campus de Ilha Solteira/ SP, UNESP, utilizando-se cinco lotes de sementes de duas cultivares de rúcula, 'Cultivada' e 'Gigante', provenientes da empresa Isla. As sementes foram submetidas à determinação do teor de água e aos testes de germinação, primeira contagem de germinação, emergência e variações na condução do teste de deterioração controlada.

As sementes estavam em recipientes herméticos do tipo lata e, durante todo o período experimental, permaneceram armazenadas em câmara seca a temperatura de $20^{\circ} \mathrm{C}$ e $50 \%$ de umidade relativa do ar.

A determinação do teor de água foi feita através do método da estufa a $105 \pm 3^{\circ} \mathrm{C}$ durante 24 horas (BRASIL, 2009), em duas subamostras com aproximadamente $2,0 \mathrm{~g}$ de sementes para cada lote. Para o teste de germinação, foram utilizadas quatro subamostras de 50 sementes por lote, distribuídas sobre uma folha de papel germitest, previamente umedecida com água destilada, na quantidade equivalente a 2,5 vezes o peso do substrato seco, colocadas no interior de caixas plásticas transparentes $(11,5 \times 11,5 \times 3,5 \mathrm{~cm})$ e mantidas em germinador regulado a temperatura constante de $25^{\circ} \mathrm{C}$. As avaliações foram realizadas aos quatro e sete dias após a semeadura (BRASIL, 2009). A primeira contagem de germinação foi realizada juntamente com o teste de germinação, computandose a porcentagem média de plântulas normais, obtidas aos quatro dias após a semeadura. Para a emergência de plântulas, foram utilizadas bandejas de isopor com 128 células individuais, contendo substrato comercial Plantmax ${ }^{\circledR}$, as quais foram mantidas em casa de vegetação com controle de temperatura $\left(25^{\circ} \mathrm{C}\right)$, com regas diárias (três vezes ao dia). Para cada lote de sementes, foram utilizadas quatro subamostras de 50 sementes, colocando-se uma semente por célula. A avaliação da emergência das plântulas foi efetuada aos 10 dias após a semeadura, mediante a contagem de plântulas normais emergidas. 
$\mathrm{Na}$ a realização do teste de deterioração controlada, o teor de água das sementes foi ajustado para 18, 21 e 24\% através do método da atmosfera úmida (ROSSETO et al., 1995). Para isso, foram colocados $40 \mathrm{~mL}$ de água em caixas plásticas transparentes $(11,5 \times 11,5 \times 3,5 \mathrm{~cm})$, com suportes para apoio de uma tela metálica, sendo colocados sobre esta uma camada de tule e distribuídas aproximadamente 5,0g de sementes, em camada uniforme, para cada lote. As caixas foram tampadas e mantidas em germinador a temperatura constante de $20^{\circ} \mathrm{C}$. Durante o umedecimento artificial, o teor de água de cada amostra foi monitorado mediante pesagens sucessivas até se obter os valores desejados. Após a obtenção do teor de água, cada amostra foi colocada em embalagem aluminizada, fechada hermeticamente e mantida por cinco dias em câmara fria $\left(8 \mathrm{a} 10^{\circ} \mathrm{C}\right)$, com a finalidade de atingir o equilíbrio higroscópico. Após esse período, as sementes foram mantidas em banho-maria a $45^{\circ} \mathrm{C}$, durante 24 e 48 horas. Em seguida, os recipientes foram imersos rapidamente em água fria para reduzir a temperatura, sendo posteriormente instalado o teste de germinação (POWELL, 1995).

As contagens foram realizadas aos quatro dias após a semeadura, computando-se a porcentagem média de plântulas normais de cada lote. Também foi determinado o teor de água das sementes após o período em banho-maria, conforme os critérios estabelecidos pelas Regras para Análise de Sementes (BRASIL, 2009).

O experimento foi montado no delineamento inteiramente casualizado e os resultados foram submetidos à análise de variância, sendo que as médias foram comparadas pelo teste de Tukey a $5 \%$ de probabilidade.

\section{RESULTADOS E DISCUSSÃO}

Pelos resultados da avaliação inicial da qualidade fisiológica dos lotes de sementes de rúcula presentes na tabela 1 , verificou-se que houve diferença significativa entre os lotes em todas as variáveis avaliadas. Para a cultivar 'Cultivada', observa-se que o lote 4 foi aquele com os menores percentuais de germinação e vigor inferior, avaliado pela primeira contagem e emergência de plântulas, sendo portanto, o de menor qualidade fisiológica. O lote 1 foi o de melhor qualidade fisiológica, não diferindo dos lotes 2,3 e 5 na germinação e lotes 3 e 5 na primeira contagem e emergência de plântulas.

Com relação à cultivar 'Gigante' (Tabela 1), não houve diferença significativa entre os lotes de sementes para o teste de germinação. Porém, resultados elevados e semelhantes no teste de germinação não significam necessariamente que todos os lotes possuem alto vigor, uma vez que o teste de germinação é conduzido em condições favoráveis de temperatura, umidade e luminosidade, permitindo ao lote a expressão do potencial máximo para produzir plântulas normais (MARCOS FILHO, 1999). Nesse sentido, os testes de primeira contagem de germinação e emergência de plântulas foram mais sensíveis, indicando a menor qualidade fisiológica dos lotes 8 e 9 em comparação aos demais.

De maneira semelhante ao teste de primeira contagem de germinação, o teste de emergência de plântulas permitiu classificar, para as duas cultivares, os lotes de melhor e pior desempenho. O teste de emergência de plântulas constitui uma variável que indica a eficiência dos testes para avaliação do potencial fisiológico de lotes de sementes (MARCOS FILHO, 1999).

Tabela 1 - Qualidade inicial de cinco lotes de sementes de rúcula das cultivares 'Cultivada' e 'Gigante'.

\begin{tabular}{|c|c|c|c|c|c|}
\hline Cultivares & Lotes & Umidade & Germinação & Primeira Contagem & Emergência \\
\hline \multirow{5}{*}{ 'Cultivada' } & 1 & 5,5 & $98 \mathrm{a}$ & $96 \mathrm{a}$ & $98 \mathrm{a}$ \\
\hline & 2 & 5,0 & $93 \mathrm{a}$ & $82 \mathrm{~b}$ & $89 \mathrm{~b}$ \\
\hline & 3 & 5,4 & $97 \mathrm{a}$ & $94 \mathrm{a}$ & $96 \mathrm{a}$ \\
\hline & 4 & 5,5 & $85 \mathrm{~b}$ & $73 \mathrm{c}$ & $78 \mathrm{c}$ \\
\hline & 5 & 5,0 & $96 \mathrm{a}$ & $95 \mathrm{a}$ & $97 \mathrm{a}$ \\
\hline \multirow{7}{*}{ 'Gigante' } & $\mathrm{CV}(\%)$ & - & 3,3 & 4,0 & 6,2 \\
\hline & 6 & 5,5 & $96 \mathrm{a}$ & $96 \mathrm{a}$ & $96 \mathrm{a}$ \\
\hline & 7 & 5,9 & $98 \mathrm{a}$ & $84 \mathrm{~b}$ & $89 \mathrm{~b}$ \\
\hline & 8 & 5,7 & $94 \mathrm{a}$ & $72 \mathrm{c}$ & $75 \mathrm{c}$ \\
\hline & 9 & 5,3 & $94 \mathrm{a}$ & $73 c$ & $76 \mathrm{c}$ \\
\hline & 10 & 5,3 & $97 \mathrm{a}$ & $95 \mathrm{a}$ & $98 \mathrm{a}$ \\
\hline & $\mathrm{CV}(\%)$ & - & 2,9 & 3,8 & 5,8 \\
\hline
\end{tabular}

* Letras distintas dentro de cada coluna diferem entre si pelo Teste de Tukey, a 5\% de probabilidade.

Ciência Rural, v.41, n.12, dez, 2011. 
Para o teste de deterioração controlada (Tabela 2), verificou-se que, de forma geral, a maioria das combinações indicaram o lote 3 da cultivar 'Cultivada' como o de menor vigor, com exceção de 48 horas a 18 e $21 \%$ de umidade, contrariando os resultados obtidos na avaliação inicial dos lotes de sementes (Tabela 1), que indicaram o lote 4 como o de menor potencial fisiológico. No condicionamento por 24 horas, o lote 1 foi o de maior vigor, não diferindo estatisticamente dos lotes 4 e 5 a $18 \%$ de umidade e do lote 4 , a $21 \%$ de umidade. Quando se utilizaram 48 horas de condicionamento, o lote 1 não diferiu estatisticamente dos lotes 4 e 5 , a 18 e $24 \%$ de umidade e dos lotes 2,4 e 5 , a $21 \%$ de umidade.

Para a cultivar 'Gigante', verificou-se que as sementes do lote 10 foram consideradas como as de menor vigor quando o teor de água foi ajustado para $24 \%$ e mantidas por 24 horas nas condições do teste de deterioração, enquanto às 48 horas os lotes 9 e 10 foram os menos vigorosos. Os lotes 6, 7 e 8 foram apontados como os de melhor qualidade fisiológica quando as sementes foram mantidas nas condições de deterioração durante 48 horas. No período de 24 horas, houve diferença significativa apenas quando o teor de água foi ajustado para $24 \%$, contrariando também os resultados obtidos na tabela 1 , ou seja, na avaliação inicial dos lotes de sementes, pois os testes iniciais indicaram os lotes 8 e 9 como os de menor vigor e 6 e 10 de maior qualidade fisiológica.

Apesar dessa diferença nos resultados obtidos, observou-se que as combinações $24 \% / 24$ e 48 horas foram as melhores para discriminar a qualidade dos lotes com relação ao potencial fisiológico. Quando o teor de água foi ajustado para $24 \%$, o teste de deterioração controlada, de forma geral, foi mais severo, causando diminuição acentuada na germinação das sementes, provavelmente, devido aos teores de umidade atingidos pelas sementes terem sido mais elevados e terem contribuído para intensificar as atividades metabólicas das sementes. POWELL \& MATHEWS (1981) também encontraram resultados semelhantes em sementes de nabo, utilizando o teste de deterioração controlada. No entanto, o ajuste do teor de água para 18 e $21 \%$ proporcionaram resultados menos drásticos.

Esses resultados estão de acordo com diversos trabalhos em sementes de hortaliças, como os de OSMAN \& GEORGE (1988) e PANOBIANCO \& MARCOS FILHO (1998) em sementes de pimentão; ISTA (1995) em sementes de beterraba e cenoura; STRYDOM \& VAN DE VENTER (1998) em sementes de repolho; ePANOBIANCO \& MARCOS FILHO (2001 a e b) em sementes de tomate. Esses autores afirmaram que o ajuste do teor de água das sementes para $24 \%$ foi o mais adequado para a condução do teste. Por outro lado, em sementes de pepino, BHERING et al. (2000) verificaram que esse teor de água foi eficiente na condução do teste de deterioração controlada, quando as sementes foram submetidas ao período de 48 horas de condicionamento em banho-maria.

$\mathrm{O}$ teor de água das sementes se manteve aproximadamente constante no decorrer do teste de deterioração controlada (Tabela 3), fato este que é um

Tabela 2 - Dados médios obtidos para o teste de deterioração controlada de cinco lotes de sementes de rúcula das cultivares 'Cultivada' e 'Gigante'.

\begin{tabular}{|c|c|c|c|c|c|c|c|}
\hline \multirow{2}{*}{ Cultivares } & \multirow{2}{*}{ Lotes } & \multicolumn{3}{|c|}{ - } & \multicolumn{3}{|c|}{ - } \\
\hline & & $18 \%$ & $21 \%$ & $24 \%$ & $18 \%$ & $21 \%$ & $24 \%$ \\
\hline \multirow{6}{*}{ 'Cultivada' } & & 07 & 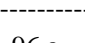 & - & 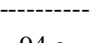 & - & 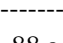 \\
\hline & 1 & $97 \mathrm{a}$ & $96 \mathrm{a}$ & $95 \mathrm{a}$ & $94 \mathrm{a}$ & $92 \mathrm{a}$ & $88 \mathrm{a}$ \\
\hline & 2 & $85 \mathrm{~b}$ & $83 \mathrm{~b}$ & $86 \mathrm{~b}$ & $82 \mathrm{~b}$ & $88 \mathrm{ab}$ & $70 \mathrm{~b}$ \\
\hline & 3 & $76 \mathrm{c}$ & $72 \mathrm{c}$ & $68 \mathrm{~d}$ & $83 \mathrm{~b}$ & $80 \mathrm{~b}$ & $64 c$ \\
\hline & 4 & $96 \mathrm{a}$ & $94 \mathrm{a}$ & $88 \mathrm{~b}$ & $88 \mathrm{ab}$ & $87 \mathrm{ab}$ & $86 a$ \\
\hline & 5 & $92 a b$ & $78 \mathrm{bc}$ & $74 \mathrm{c}$ & $92 \mathrm{a}$ & $88 \mathrm{ab}$ & $85 \mathrm{a}$ \\
\hline \multirow{7}{*}{ 'Gigante' } & $\mathrm{CV}(\%)$ & \multicolumn{6}{|c|}{4,2} \\
\hline & 6 & $98 \mathrm{a}$ & 97 a & $98 \mathrm{a}$ & $98 \mathrm{a}$ & $94 \mathrm{a}$ & $96 \mathrm{a}$ \\
\hline & 7 & $97 \mathrm{a}$ & $96 a$ & $96 \mathrm{a}$ & $97 \mathrm{a}$ & $96 \mathrm{a}$ & $94 \mathrm{a}$ \\
\hline & 8 & $98 \mathrm{a}$ & $97 \mathrm{a}$ & $98 \mathrm{a}$ & $97 \mathrm{a}$ & $95 \mathrm{a}$ & $96 a$ \\
\hline & 9 & $93 \mathrm{ab}$ & $93 \mathrm{ab}$ & $91 \mathrm{~b}$ & $88 \mathrm{~b}$ & $86 \mathrm{~b}$ & $81 \mathrm{~b}$ \\
\hline & 10 & $85 \mathrm{~b}$ & $82 \mathrm{~b}$ & $80 \mathrm{c}$ & $84 \mathrm{~b}$ & $82 \mathrm{~b}$ & $78 \mathrm{c}$ \\
\hline & $\mathrm{CV}(\%)$ & \multicolumn{6}{|c|}{4,4} \\
\hline
\end{tabular}

*Letras iguais dentro de cada coluna não diferem entre si pelo teste de Tukey a 5\% de probabilidade.

Ciência Rural, v.41, n.12, dez, 2011. 
Tabela 3 - Dados médios obtidos para teor de água após o teste de deterioração controlada de cinco lotes de sementes de rúcula das cultivares 'Cultivada' e 'Gigante'.

\begin{tabular}{|c|c|c|c|c|c|c|c|}
\hline \multirow[b]{2}{*}{ Cultivares } & \multirow[b]{2}{*}{ Lotes } & \multicolumn{3}{|c|}{ - } & \multicolumn{3}{|c|}{ 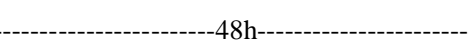 } \\
\hline & & 18 & 21 & 24 & 18 & 21 & 24 \\
\hline \multirow{6}{*}{ 'Cultivada' } & & - & - & 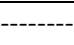 & ------- & ----- & --1---- \\
\hline & 1 & 18,1 & 21,0 & 24,2 & 18,1 & 21,2 & 24,0 \\
\hline & 2 & 18,0 & 21,2 & 24,0 & 17,9 & 21,0 & 24,2 \\
\hline & 3 & 17,9 & 21,1 & 24,1 & 18,2 & 20,9 & 24,1 \\
\hline & 4 & 18,2 & 20,9 & 24,2 & 18,2 & 21,2 & 23,9 \\
\hline & 5 & 18,3 & 21,1 & 23,9 & 18,0 & 21,1 & 24,0 \\
\hline \multirow{5}{*}{ 'Gigante' } & 6 & 17,9 & 21,3 & 24,2 & 18,0 & 21,1 & 24,1 \\
\hline & 7 & 18,2 & 21,2 & 24,1 & 18,1 & 21,0 & 24,0 \\
\hline & 8 & 18,0 & 21,0 & 24,1 & 18,0 & 20,9 & 23,9 \\
\hline & 9 & 18,1 & 20,9 & 24,0 & 18,2 & 21,2 & 24,0 \\
\hline & 10 & 18,0 & 21,3 & 24,2 & 18,2 & 21,0 & 24,2 \\
\hline
\end{tabular}

pré-requisito para a sua condução (MATTHEWS, 1980). O uso da embalagem aluminizada foi eficiente para a manutenção da umidade desejada, porém não se observou relação entre os resultados obtidos para o teste de deterioração controlada e as avaliações iniciais do potencial fisiológico das sementes, principalmente para o teste de emergência em casa de vegetação.

\section{CONCLUSÃO}

O teste de deterioração controlada, nas condições testadas, não é eficiente na avaliação do potencial fisiológico de sementes de rúcula das cultivares 'Cultivada' e 'Gigante', quando comparado ao teste de emergência de plântulas.

\section{REFERÊNCIAS}

BHERING, M.C. et al. Métodos para avaliação do vigor de sementes de pepino. Revista Brasileira de Sementes, v.22, n.2, p.171175, 2000. Disponível em: <http://www.scielo.br/ scielo.php?pid=S0101-31222005000100025\&script=sci_arttext $>$. Acesso em 10 jan. 2011. doi: 10.1590/S0101-31222005000100024.

BHERING, M.C. et al. Testes para avaliação da qualidade fisiológica de sementes de berinjela. Informativo ABRATES, v.11, n.2, p.203, 2001a.

BHERING, M.C. et al. Aplicação do vigor de sementes de melancia pelos testes de envelhecimento acelerado e deterioração controlada. Informativo ABRATES, v.11, n.2, p.204, 2001b.

BRASIL. Ministério da Agricultura, Pecuária e Abastecimento. Regras para análise de sementes. Brasília, DF: MAPA/ ACS, Secretaria de Defesa Agropecuária, 2009. 399p.
HAMPTON, J.G.; TEKRONY, B.H. Conductivity test. In: HAMPTON, J.G.; TEKRONY, B.M. (Ed.). Handbook of vigor methods. 3.ed. Zürich: ISTA, 1995. p.22-34.

INTERNATIONAL SEED TESTING ASSOCIATION. Handbook of vigour test methods. 3.ed. Zürich: ISTA, 1995. 116p.

MARCOS FILHO, J. Testes de vigor: importância e utilização. In: KRZYZANOWSKI, F.C. et al. (Ed.). Vigor de sementes: conceitos e testes. Londrina: ABRATES, 1999. p.1-21.

MATTHEWS, S. Controlled deterioration: a new vigour test for crop seeds. In: HEBBLETHWAITE, P.D. (Ed.). Seed production. London: Butterworths, 1980. p.647-660.

MATTHEWS, S.; POWELL, A.A. Controlled deterioration test. In: PERRY, D.A. (Ed.). Handbook of vigour test methods. 2.ed. Zürich: ISTA, 1987. p.49-56.

MENDONÇA, E.A.F. et al. Teste de deterioração controlada em sementes de brócolis (Brassica oleracea L.) var. italica. Revista Brasileira de Sementes, v.22, n.1, p.280-287, 2000.

OLUOCH, M.O.; WELBAUM, G.E. Effect of postharvest washing and pos-storage priming on viability and vigour of sixyear-old muskmelon (Cucumis melo L.) seeds from eight stages of development. Seed Science and Technology, v.24, n.2, p.195-209, 1996.

OSMAN, O.A.; GEORGE, R.A.T. Controlled deterioration as a vigour test for sweet pepper seed. Acta Horticulturae, v.218, n.4, p.110-114, 1988.

PANOBIANCO, M.; MARCOS FILHO, J. Comparação de métodos para avaliação da qualidade fisiológica de sementes de pimentão. Revista Brasileira de Sementes, v.20, n.2, p.306$310,1998$.

PANOBIANCO, M.; MARCOS FILHO, J. Envelhecimento acelerado e deterioração controlada em sementes de tomate. Scientia Agrícola, v.58, n.3, p.525-531, 2001a. Disponível em: 
<http://www.scielo.br/scielo.php?script=sci_arttext\&pid=S0103$90162001000300014 \& \operatorname{lng}=$ pt\&nrm=iso\&tlng=pt $>$. Acesso em 10 jan. 2011. doi: 10.1590/S0103-90162001000300014.

PANOBIANCO, M.; MARCOS FILHO, J. Evaluation of the physiological potential of tomato seeds by germination and vigor tests. Seed Technology, v.23, n.2, p.151-161, 2001b.

POWELL, A.A.; MATTHEWS, S. Evaluation of controlled deterioration, a new vigour test for small seed vegetables. Seed Science and Technology, v.9, n.2, p.633-640, 1981.

POWELL, A.A. et al. Assessment of the repeatability of the controlled deterioration vigour test both within and between laboratories. Seed Science and Technology, v.12, n.2, p.421427, 1984.

POWELL, A.A. The controlled deterioration test. In: VAN DE VENTER, H.A. (Ed.). Seed vigourtesting seminar. Copenhagen: ISTA, 1995. p.73-87.

POWELL, A.A. et al. Identification of vigour differences among combining pea (Pisum sativum) seed lots. Seed Science and Technology, v.25, n.3, p.443-464, 1997.

PURQUERIO, L.F.V. Crescimento, produção e qualidade de rúcula (Eruca sativa Miller) em função do nitrogênio e da densidade de plantio. 2005. 138f. Tese (Doutorado em Horticultura) - Faculdade de Ciências Agronômicas, Universidade Estadual Paulista, Botucatu, SP.

RODO, A.B. et al. Teste de condutividade elétrica em sementes de tomate. Revista Brasileira de Sementes, v.20, n.1, p.29-38, 1998

ROSSETO, C.A.V. et al. Metodologias de ajuste do teor de água e comportamento das sementes de soja no teste de germinação. Revista Brasileira de Sementes, v.17, n.2, p.171-178, 1995.

SADER, R. et al. Teste de deterioração controlada em sementes de brocoli (Brassica oleracea L. var. italica). Informativo ABRATES, v.11, n.2, p.175, 2001.

STRYDOM, A.; VAN DE VENTER, H.A. Comparison of seed vigour tests for cabbage (Brassica oleracea $\mathrm{L}$. var. capitata). Seed Science and Technology, v.26, n.3, p.579-585, 1998.

TORRES, S.B. et al. Testes de vigor em sementes de maxixe (Cucumis anguria L.) com ênfase ao teste de condutividade elétrica. Revista Brasileira de Sementes, v.20, n.2, p.480483, 1998.

ZHANG, T.; HAMPTON, J.G. Does fungicide seed treatment affect bulk conductivity test results? Seed Science and Technology, v.27, n.3, p.1041-1045, 1999. 